\title{
Hypofractionated stereotactic radiotherapy for dumbbell-shaped hypoglossal schwannomas: Two cases of long-term follow-up and a review of the literature
}

\author{
YONG LI ${ }^{1}$, JINRONG LOU ${ }^{2}$, SHUJUN QIU ${ }^{1}$, YUTIAN GUO ${ }^{1}$ and MIANSHUN PAN ${ }^{1}$ \\ ${ }^{1}$ Center of Radiation Oncology, Wujing Hospital, Shanghai 201103; \\ ${ }^{2}$ Department of Internal Medicine, Jiading Central Hospital, Shanghai 201800, P.R. China
}

Received July 3, 2015; Accepted June 2, 2016

DOI: $10.3892 /$ mco.2016.922

\begin{abstract}
Cases of hypoglossal schwannoma are extremely rare. Historically, microsurgical resection has been the standard treatment, but it may not always be feasible; thus, it is crucial to investigate alternative treatments. We herein present the cases of two patients, both of whom presented with tongue deviation and hemiatrophy, accompanied by headaches. Magnetic resonance imaging revealed a dumbbell-shaped tumor originating from the hypoglossal nerve that was adjacent to the cranial base in each patient. Hypofractionated stereotactic radiotherapy was used to treat the tumors, with a total dose of 30 Gy in 3-Gy fractions delivered to the planning target volume. Several months later, the tumors had significantly decreased in size and the symptoms of the two patients had gradually improved.
\end{abstract}

\section{Introduction}

Microsurgical resection has been the standard treatment for hypoglossal schwannoma, but it may not always be feasible, particularly when preservation of neurological function is desired. Moreover, resection may be associated with unintended neurological deficits and other potentially severe complications $(1,2)$. Therefore, further investigation is required to design a safe and effective treatment for hypoglossal schwannomas. In this study, we report our experience with treating hypoglossal schwannomas using hypofractionated stereotactic radiotherapy.

\section{Case reports}

Case 1. A 33-year-old woman presented with a 2-month history of intermittent right occipital headache; in addition, her

Correspondence to: Dr Mianshun Pan, Center of Radiation Oncology, Wujing Hospital, 380 Hongxu Road, Shanghai 201103, P.R. China

E-mail: panmianshun@126.com

Key words: hypoglossal Schwannoma, stereotactic radiotherapy already existing neck rotation disorder, unclear pronunciation and dysphagia had gradually worsened over $\sim 3$ months. On neurological examination, the patient was alert and oriented. The cranial nerve functions were nearly intact, with the exception of a tongue deviation to the right side and accompanying hemiatrophy. The muscle strength and tone in all the extremities were normal. The examination for neurofibromatosis type 2 was negative. The magnetic resonance imaging (MRI) scan revealed a cystic mass adjacent to the cranial base and extending into the right hypoglossal canal. The tumor was dumbbell-shaped, measured $20 \times 18 \times 42 \mathrm{~mm}$, with distinct borders. A contrast-enhanced MRI showed diffuse enhancement in the solid portion of the tumor. The clinical diagnosis was hypoglossal schwannoma. As the patient refused surgery due to concerns regarding the risks of the operation, stereotactic radiotherapy (SGS-I Stereotactic Gamma-Ray system; Huiheng Medical Inc., Shenzhen, China) was used to treat the tumor. The gross tumor volume (GTV) included the intracranial and extracranial lesions. A margin of $2 \mathrm{~mm}$ was added to the GTV to formulate the planning target volume (PTV). A dose of $30 \mathrm{~Gy}$ in fraction sizes of $3 \mathrm{~Gy}$ was delivered to the PTV. The edges of the PTV and GTV were encompassed by $50 \%$ and $70 \%$ isodose curves, respectively. The maximum dose to the spinal cord was 25 Gy. Six months after the treatment, the patient experienced gradual improvement of the symptoms. The MRI scan performed 5 years after the stereotactic radiotherapy revealed that the hypoglossal neurinoma exhibited a complete response. The patient's headaches had disappeared and her neck movements had become smooth; her pronunciation and eating ability had also significantly improved (Fig. 1).

Case 2. A 38-year-old man presented with throbbing left occipital headache that had persisted for $\sim 6$ months. The pain was severe at night and affected his ability to sleep. A physical examination revealed left tongue hemiatrophy, without any clinical manifestations of neurofibromatosis type 2. The MRI scan revealed a dumbbell-shaped cystic mass adjacent to the cranial base that extended into the left hypoglossal canal and measured $\sim 25 \times 20 \times 48 \mathrm{~mm}$. A contrast-enhanced MRI showed obvious enhancement in the solid portion of the tumor. The tumor was ultimately diagnosed as hypoglossal schwannoma. 

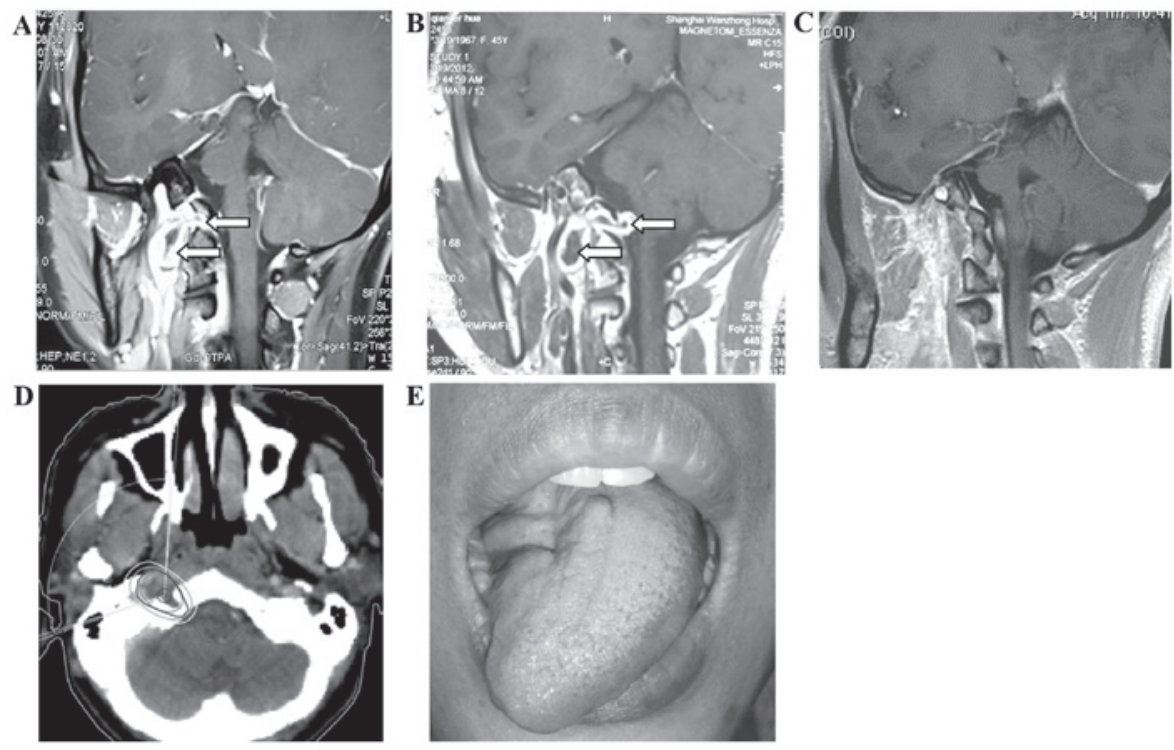

Figure 1. Follow-up images of case 1. (A) Prior to radiotherapy, the tumor (arrow) on the right hypoglossal was clearly enhanced. (B) The tumor was reduced in volume and exhibited necrosis (arrow) 6 months after radiotherapy. (C) The lesion had disappeared 5 years after radiotherapy. (D) Radiotherapy treatment planning for the tumor. (E) The tongue of the patient deviated to the right side and exhibited hemiatrophy prior to radiotherapy.
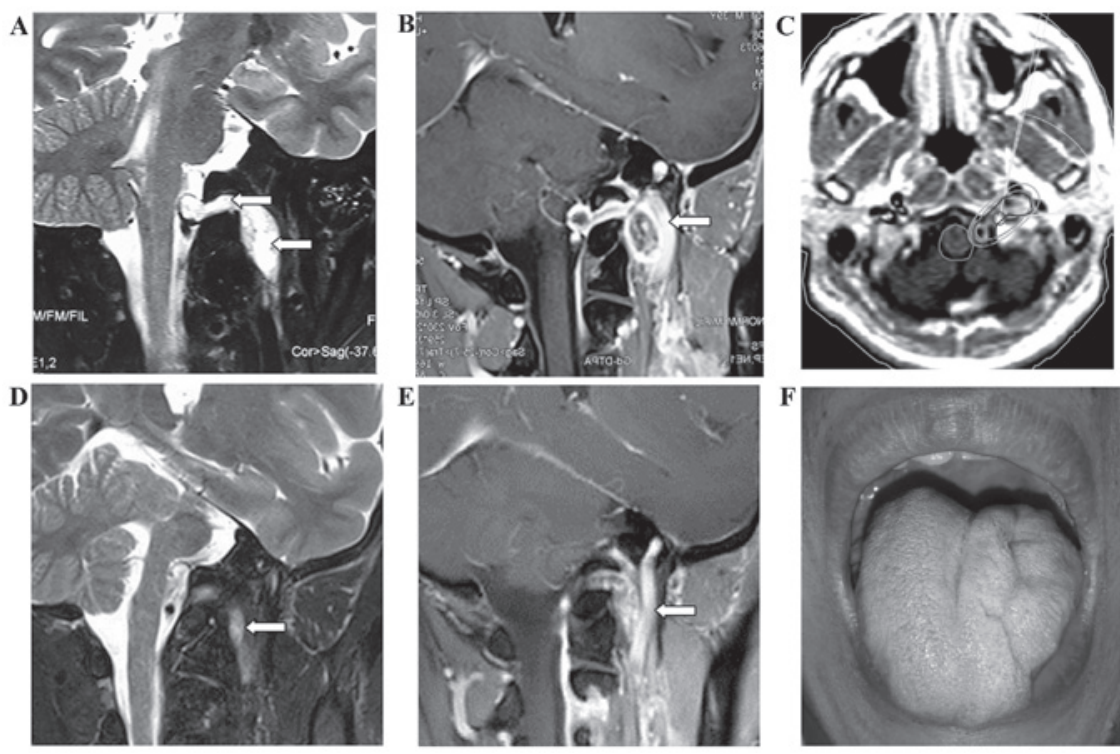

Figure 2. Follow-up images of case 2. (A) Dumbbell-shaped hypoglossal schwannoma (arrow) extending intracalvarially through the left hypoglossal canal (B) The left internal carotid artery and the vertebral artery (arrow) were compressed by the tumor. (C) Radiotherapy planning for the intracranial and extracranial lesions. (D) The tumor (arrow) had distinctly decreased in size 4 years after the treatment. (E) The vascular (arrow) compression was relieved. (F) Left side of the tongue exhibiting hemiatrophy prior to radiotherapy.

The patient refused surgery and instead opted for stereotactic radiotherapy. A total dose of $30 \mathrm{~Gy}$ in 10 fractions was delivered to the intracranial and extracranial lesions, with a maximum dose to the spinal cord of $26 \mathrm{~Gy}$. Three months after the treatment, the patient's headaches had gradually improved. The MRI scan performed 4 years after the treatment revealed that the hypoglossal neurinoma had decreased in size, and the patient's headaches had disappeared (Fig. 2).

\section{Discussion}

The hypoglossal nerve, which is a motor nerve, arises from the hypoglossal nucleus in the brainstem and, after passing through the subarachnoid space, it exits the skull base of the posterior fossa through the hypoglossal canal. Upon emerging from the hypoglossal canal, the hypoglossal nerve spirals behind the vagus nerve and passes between the internal carotid artery and the internal jugular vein lying on the carotid sheath. After passing deep into the posterior belly of the digastric muscle, it reaches and innervates the ipsilateral tongue muscles.

Hypoglossal schwannomas are benign tumors originating from the Schwann cells of the hypoglossal nerve. These tumors are extremely rare, constituting only $\sim 1 \%$ of all intracranial schwannomas, as neurinomas develop more often in sensory rather than motor nerves (3). To the best of our knowledge, a total of 89 cases have been reported in the English literature 
over the last three decades. Among those cases, the male:female ratio was 2:3 and Asian patients accounted for $73.0 \%$ of the cases $(29.2 \%$ were from from China, $23.6 \%$ from Japan and $16.8 \%$ from India). The patients were aged $9-85$ years, with the highest incidence between 30 and 60 years (73.3\%).

Hypoglossal schwannomas may be classified according to tumor location as follows: Type A, intracranial tumor, accounting for $31.5 \%$ of the cases; type B, both intra- and extracranial tumor (referred to as dumbbell-shaped tumor), accounting for $50.0 \%$ of the cases; and type C, extracranial-only tumor, accounting for $18.5 \%$ of the cases (4). There is a variety of clinical symptoms associated with the progress of hypoglossal schwannomas, as the growth of the tumor compresses the vessels and nerves adjacent to it. The most common symptom is hypoglossal nerve palsy (93.5\%). Other symptoms include palsies of the lower cranial nerves $(50 \%)$ such as the glossopharyngeal and vagus nerves; cerebellar signs (47.8\%); occipital and nuchal pain (54.3\%) due to radicular and meningeal irritation; and long-term signs, such as motor $(41.3 \%)$ and sensory disturbances $(37.0 \%)$ due to compression by the tumor (5). In the most common schwannomas, two distinct histological patterns are observed, referred to as Antoni types A and B. Antoni type A tissues are characterized by compact Schwann cells with nuclear palisading, whereas Antoni type B tissues display considerable cell pleomorphism in a loosely arranged reticular network, often with cystic spaces (6). The cystic spaces may result in high signal intensity on T2-weighted MRI images. Schwannomas are typically isointense or mildly hypointense relative to the gray matter on T1-weighted images. Gadolinium enhancement is typically homogeneous, whereas some larger schwannomas may include areas of cystic degeneration and heterogeneous signal intensity; these findings are based on increased numbers of areas with Antoni type B histological characteristics. In our two cases, the tumors were dumbbell-shaped, with intra- and extracranial components, and were both associated with the typical symptoms and imaging characteristics of a hypoglossal schwannoma.

As hypoglossal schwannomas are benign, slow-growing tumors, complete resection may be curative. However, microsurgical resection has traditionally been associated with a high risk of postoperative deficits and mortality, particularly in cases of dumbbell-shaped hypoglossal schwannomas. Over $50 \%$ of these patients succumbed due to respiratory distress within 4 weeks after surgery (7). However, a better understanding of the cranial base microanatomy and the operative approaches has led to the development of the extreme lateral infrajugular transcondylar-transtubercular exposure (ELITE) technique. This approach provides sufficient exposure for the majority of dumbbell-shaped hypoglossal schwannomas $(8,9)$. Nonaka et al (10) reported 13 cases of hypoglossal schwannomas that were managed with the ELITE technique, which is the largest series to date; a gross total resection of the tumor was achieved in 10 patients, a near-total tumor resection in 1 patient, and a subtotal resection in 2 patients. However, there was no improvement in the preoperative cranial nerve deficits in any of the patients following surgery. Postoperative hoarseness was observed in 4 patients (30.7\%), dysphagia in $3(23.1 \%)$, facial weakness in $1(7.7 \%)$ and cerebrospinal fluid leaks, which persisted despite the use of lumbar drains, in 1 patient $(7.7 \%)$. The results demonstrated that, although surgery may achieve maximum tumor removal, the benefits for patients may not be optimal. In the modern era, intracranial schwannomas are detected at an earlier stage; consequently, they are usually of small to moderate size at diagnosis, and are therefore amenable to radiosurgery, which offers a minimally invasive alternative to microsurgery. Although the literature reports involve a small number of patients, they shed some light on the effectiveness and safety of radiosurgery for the treatment of hypoglossal schwannoma. Kimball et al (11) reported one case treated with linear accelerator radiosurgery, in which the patient experienced an improvement in dysphonia. In addition, Elsharkawy et al (12) reported two cases of hypoglossal schwannoma that regressed following treatment with Gamma Knife radiosurgery.

Fractionated radiotherapy has been used in certain centers for the treatment of non-vestibular schwannomas and has achieved tumor control similar to that achieved with radiosurgery. Showalter et al (13) reported a series of patients who received radiotherapy for non-acoustic cranial nerve schwannomas (including two cases of hypoglossal schwannomas); a total of 24 patients received fractionated radiotherapy, delivered in 1.8- to 2.0-Gy fractions at a median dose of $50.4 \mathrm{~Gy}$; 15 patients received radiosurgery at a median dose of $12.0 \mathrm{~Gy}$. The 2-year actuarial tumor control rate following fractionated radiotherapy and radiosurgery was $95 \%$. Cranial nerve deficits improved in $50 \%$, remained stable in $46 \%$, and worsened in $4 \%$ of the patients. No significant difference was observed between fractionated radiotherapy and radiosurgery in terms of local control or improvement in the cranial nerve-related symptoms.

To the best of our knowledge, hypofractionated stereotactic radiotherapy has not been previously reported in the treatment of hypoglossal schwannoma. The two cases reported herein demonstrate that this technique is safe and effective. The total dose of 30 Gy in $103-$ Gy fractions may be the optimal dosage.

Microsurgical resection remains an important tool in the treatment of hypoglossal schwannoma, particularly for patients with giant tumors or those with more severe symptoms. However, hypofractionated stereotactic radiotherapy or radiosurgery may be a more valuable treatment option for patients with small tumors or those with milder symptoms, and it is particularly suitable for postoperative residual lesions.

\section{References}

1. Sarma S, Sekhar LN and Schessel DA: Nonvestibular schwannomas of the brain: A 7-year experience. Neurosurgery 50: 437-448; discussion 438-439, 2002.

2. Nishioka K, Abo D, Aoyama H, Furuta Y, Onimaru R, Onodera S, Sawamura Y, Ishikawa M, Fukuda S and Shirato H: Stereotactic radiotherapy for intracranial nonacoustic schwannomas including facial nerve schwannoma. Int $\mathrm{J}$ Radiat Oncol Biol Phys 75: 1415-1419, 2009.

3. Heiroth HJ, Riemenschneider MJ, Steiger HJ and Hänggi D: A cylindrical extracranial cranial base neurinoma of the hypoglossal nerve: A rare tumor with a rare localization: Case report. Neurosurgery 65: E212-E213; discussion E213, 2009.

4. Hoshi M, Yoshida K, Ogawa K and Kawase T: Hypoglossal neurinoma - two case reports. Neurol Med Chir (Tokyo) 40: 489-493, 2000

5. Sato M, Kanai N, Fukushima Y, Matsumoto S, Tatsumi C, Kitamura K, Ozaki M and Hayakawa T: Hypoglossal neurinoma extending intra- and extracranially: Case report. Surg Neurol 45: $172-175,1996$. 
6. Jadwani S, Bansod S and Mishra B: Intraoral schwannoma in retromolar region. J Maxillofac Oral Surg 11: 491-494, 2012.

7. Williams JM and Fox JL: Neurinoma of the intracranial portion of the hypoglossal nerve. Review and case report. J Neurosurg 19: 248-250, 1962.

8. Sen CN and Sekhar LN: An extreme lateral approach to intradural lesions of the cervical spine and foramen magnum. Neurosurgery 27: 197-204, 1990.

9. Bertalanffy $\mathrm{H}$ and Seeger W: The dorsolateral, suboccipital, transcondylar approach to the lower clivus and anterior portion of the craniocervical junction. Neurosurgery 29: 815-821, 1991.

10. Nonaka Y, Grossi PM, Bulsara KR, Taniguchi RM, Friedman AH and Fukushima T: Microsurgical management of hypoglossal schwannomas over 3 decades: A modified grading scale to guide surgical approach. Neurosurgery 69 (2 Suppl Operative): ons121-140; discussion ons140, 2011.
11. Kimball MM, Foote KD, Bova FJ, Chi YY and Friedman WA: Linear accelerator radiosurgery for nonvestibular schwannomas. Neurosurgery 68: 974-984; discussion 984, 2011.

12. Elsharkawy M, Xu Z, Schlesinger D and Sheehan JP: Gamma Knife surgery for nonvestibular schwannomas: Radiological and clinical outcomes. J Neurosurg 116: 66-72, 2012.

13. Showalter TN, Werner-Wasik M, Curran WJ Jr, Friedman DP, $\mathrm{Xu} \mathrm{X}$ and Andrews DW: Stereotactic radiosurgery and fractionated stereotactic radiotherapy for the treatment of nonacoustic cranial nerve schwannomas. Neurosurgery 63: 734-740, 2008. 